\title{
Development of Metal-Organic Framework (MOF) Coated Bulk Acoustic Sensors for Carbon Dioxide and Methane Detection
}

\author{
Jenna A. Gustafson ${ }^{1}$, Jagannath Devkota ${ }^{2,3}$, Christopher E. Wilmer ${ }^{1}$, Paul R. Ohodnicki ${ }^{2}$ \\ 1 University of Pittsburgh Department of Chemical \& Petroleum Engineering, \\ 3700 O'Hara St. Pittsburgh, PA 15215, USA, \\ ${ }^{2}$ National Energy Technology Laboratory, 626 Cochrans Mill Rd, Pittsburgh, PA 15236, USA \\ ${ }^{3}$ AECOM, Pittsburgh, PA 15236, USA \\ jag227@pitt.edu
}

\begin{abstract}
:
In this study, a quartz crystal microbalance (QCM) sensor coated with a thin layer of the metal-organic framework (MOF) ZIF-8 was fabricated and tested for detection of $\mathrm{N}_{2} / \mathrm{CO}_{2} / \mathrm{CH}_{4}$ with a comparison to a computational analysis. We analyzed the mass uptake of mixtures of $\mathrm{N}_{2}, \mathrm{CO}_{2}$, and $\mathrm{CH}_{4}$ in $\mathrm{ZIF}-8$ in atmospheric conditions, using all mixtures with varying each component from 0 to 100 percent by 20 percent increments. Experiments used a ZIF-8 film of $500 \mathrm{~nm}$ thickness and resulted in mass uptake of the gases showing a positive linear relationship with $\mathrm{CO}_{2}$. Similarly, the adsorption of all mixtures in the MOF were obtained computationally via grand canonical Monte Carlo (GCMC) simulations, reported also as the total mass uptake of the MOF. We directly compare our experimental and computational results, showing that similar trends exist in the affinity of $\mathrm{ZIF}-8$ towards $\mathrm{CO}_{2}$. We show that computational methods may be used to model the output of a chemical sensor, specifically in this work for a MOF on QCM device.
\end{abstract}

Key words: chemical sensor, quartz crystal microbalance, metal-organic framework, simulations, electronic nose

\section{Introduction}

Gas sensors have been used for decades, serving a range of applications from food quality assessment to environmental monitoring [1]. In particular, arrays of sensors (i.e., electronic noses) are used as an improvement upon single sensors in that their complex design allows for reduced signal-to-noise ratios and more sensitive sensors. However, even gas sensor arrays are not currently able to reach the sensitivity and selectivity required for certain applications, including the detection of some toxic chemical leaks and the detection of some diseases via breath analysis [2].

In this study we have chosen to layer a quartz crystal microbalance (QCM) device with a sensing layer of the metal-organic framework (MOF) ZIF-8, from which total frequency shifts are obtained as a result of gas mixture adsorption. QCMs are suitable candidate devices for electronic nose development, particularly due to their fast response times and portability [3]. MOFs are of particular interest as candidate sensing materials due to their highly crystalline structures which have record high adsorption capacities and are able to be tuned for specific gas components [4]. We report experimental and simulation results for a ZIF-8 coated sensor detecting various mixtures of $\mathrm{CO}_{2}, \mathrm{CH}_{4}$, and $\mathrm{N}_{2}$.

\section{Methodology}

\subsection{Experimental}

The procedures for ZIF-8 sensing layer coating and gas test measurements can be found elsewhere [5]. Briefly, gold-coated AT-cut quartz crystal (crystal diameter $25.4 \mathrm{~mm}$, and piezoelectrically active area $=34.19 \mathrm{~mm}^{2}$ ) resonators of resonance frequency $5 \mathrm{MHz}$ and its measuring assembly were purchased from INFICON. ZIF-8 films of $500 \mathrm{~nm}$ thickness were coated on all sides of the substrates at room temperature by five repetitive cycles of dip coating method. The gas testing measurements were performed in a $1000 \mathrm{~mL}$ gas cell (vol $~ 1000$ $\mathrm{mL}$ ) connected to an automated mass flow controller. Nitrogen was used as the carrier gas.

\subsection{Simulations}

Grand canonical Monte Carlo (GCMC) simulations were performed to obtain adsorption of various mixtures of $\mathrm{N}_{2}, \mathrm{CH}_{4}$, and $\mathrm{CO}_{2}$, of $20 \%$ increments, in ZIF-8 at $298 \mathrm{~K}$ and 1 bar. The software package RASPA was used, along with 
the universal force field (UFF) and TraPPE force field parameters to define structure atoms and gas molecules, respectively [6,7]. These simulations provide the total mass adsorbed onto the MOF upon exposure to each gas mixture. This total mass change can be converted to a frequency shift via the Sauerbrey equation (eq. (1)), used to compare with experimental QCM signals.

$$
\frac{\Delta m}{A}=\frac{C_{m}\left(f-f_{0}\right)}{2 f^{2}}
$$

In eq. (1) $\Delta m / A$ is the mass change per area, $C_{m}$ is the mechanical coefficient for quartz, $f_{0}$ is the fundamental resonance frequency in reference state $(5 \mathrm{MHz})$, and $\mathrm{f}$ is the output frequency shift. Thus, we can simulate and predict a response for a MOF/QCM sensor.

\section{Results and Discussion}

Frequency shifts obtained from experiments are shown in Figure 1, along with calculated frequencies derived from simulations. The two data sets follow similar trends but are not in agreement with the magnitude of their frequency values. Discrepancies in the data may be a result of the variations in MOF material properties, such as having solvent left in its pores. Furthermore, the forcefield parameters used in simulations may not yield the best model for ZIF-8, however, this computational approach has proven accurate for predicting gas behavior in many MOFs $[8,9]$.

ZIF-8 exhibits selectivity towards $\mathrm{CO}_{2}$, while changes in $\mathrm{CH}_{4}$ and $\mathrm{N}_{2}$ are more challenging to detect. With the interest of natural gas leaks in mind, it is a difficult task to optimize detection of small $\mathrm{CH}_{4}$ concentrations. This is where arrays of sensors will be effective in reducing signal to noise ratios and finely tuning the sensing materials to sense gases of interest.

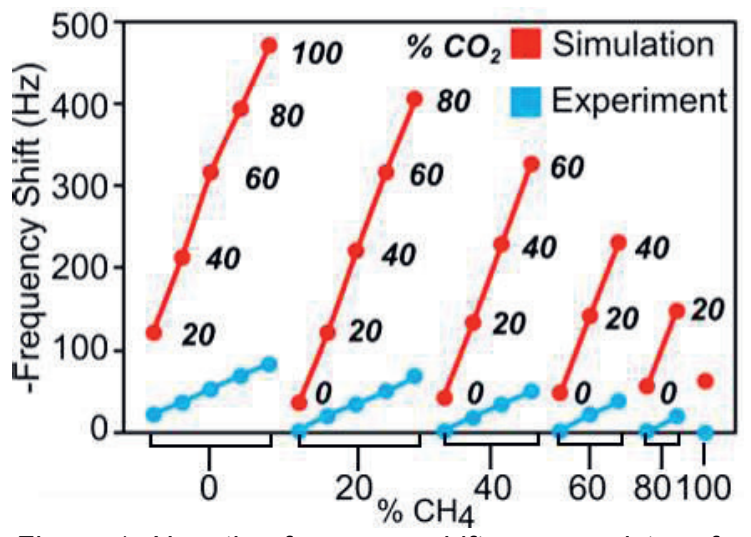

Figure 1. Negative frequency shift vs gas mixture for various concentrations of $\mathrm{N}_{2}, \mathrm{CO}_{2}$, and $\mathrm{CH}_{4}$.

\section{Conclusion}

In this work, we have shown that MOFs may be good candidate materials to integrate with acoustic devices like QCMs for gas sensing. Furthermore, computational tools are appropriate for predicting mass loading in these types of sensors and therefore may be used to develop optimal sensors for various applications. We can expand this method to surface acoustic wave devices [10], and apply the analysis with a variety of MOF materials. Eventually, the sensors will be constructed in array format and applied as electronic noses.

\section{References}

[1] A.D. Wilson, M. Baietto, Applications and Advances in Electronic-Nose Technologies, Sensors. 9 5099_ 5148 (2009); doi: 10.3390/s90705099.

[2] D. Zhang, N. Yin, B. Xia, Facile Fabrication of $\mathrm{ZnO}$ Nanocrystalline-Modified Graphene Hybrid Nanocomposite Toward Methane Gas Sensing Application, J. Mater. Sci. Mater. Electron. 26 59375945 (2015); doi: 10.1007/s10854-015-3165-2.

[3] D. James, S.M. Scott, Z. Ali, W.T. O'Hare, Chemical Sensors for Electronic Nose Systems, Microchim. Acta. 149 1-17 (2004); doi: 10.1007/s00604-0040291-6.

[4] L.E. Kreno, K. Leong, O.K. Farha, M. Allendorf, R.P. Van Duyne, J.T. Hupp, Metal-Organic Framework Materials as Chemical Sensors, Chem. Rev. 112 1105-1125 (2012); doi: 10.1021/cr200324t.

[5] J. Devkota, K.-J. Kim, P.R. Ohodnicki, J.T. Culp, D.W. Greve, J.W. Lekse, Zeolitic Imidazole Framework-Coated Acoustic Sensors for Room Temperature Detection of Carbon Dioxide and Methane, ArXiv171208468 Phys. (2017); http://arxiv.org/abs/1712.08468.

[6] M.G. Martin, J.I. Siepmann, Transferable Potentials for Phase Equilibria. 1. United-Atom Description of n-Alkanes, J. Phys. Chem. B. 102 2569-2577 (1998); doi: 10.1021/jp972543+.

[7] D. Dubbeldam, S. Calero, D.E. Ellis, R.Q. Snurr, RASPA: Molecular Simulation Software for Adsorption and Diffusion in Flexible Nanoporous Materials, Mol. Simul. 42 81-101 (2016); doi: 10.1080/08927022.2015.1010082.

[8] C.E. Wilmer, O.K. Farha, T. Yildirim, I. Eryazici, V. Krungleviciute, A.A. Sarjeant, R.Q. Snurr, J.T. Hupp, Gram-Scale, High-Yield Synthesis of a Robust Metal-Organic Framework for Storing Methane and Other Gases, Energy Environ. Sci. 6 1158-1163 (2013); doi: 10.1039/C3EE24506C.

[9] J.A. Gustafson, C.E. Wilmer, Computational Design of Metal-Organic Framework Arrays for Gas Sensing: Influence of Array Size and Composition on Sensor Performance, J. Phys. Chem. C. 121 6033-6038 (2017); doi: 10.1021/acs.jpcc.6b09740.

[10] J. Devkota, P.R. Ohodnicki, D.W. Greve, SAW Sensors for Chemical Vapors and Gases, Sensors. 17801 (2017); doi:10.3390/s17040801 